\title{
Morfometría del Esófago Abdominal y del Estómago del Conejo (Orycotolagus cuniculus). Aplicaciones a la Cirugía Laparoscópica
}

\author{
Morphometry of the Abdominal Esophagus and Stomach of the Rabbit. \\ Laparoscopyc Surgery Applications
}

"Rodríguez-Alarcón, C.; "Pérez, E.; "**Martín, U.; "Rivera, R.; **Hernández, A.; "Vivo, J.; "Beristain, M. \& "Usón, J.

\begin{abstract}
RODRÍGUEZ-AlARCón, C.; PÉREZ, E.; MARTÍN, U.; RIVERA, R.; HERNÁNDEZ, A.; VIVO, J.; BERISTAIN, M. \& USÓN, J. Morfometría del esófago abdominal y del estómago del conejo (Oryctolagus cuniculus). Aplicaciones a la cirugía laparoscópica. Int. J. Morphol., 28(1):27-31, 2010.
\end{abstract}

RESUMEN: Actualmente existen pocos estudios sobre la anatomía y fisiología del tracto digestivo del conejo (Orycotolagus cuniculus), estos estudios están encaminados a la nutrición y biología del conejo, pero pocos abordan la anatomía con la visión de un cirujano. Por otro lado existen algunos estudios que proponen al conejo como modelo animal para cirugías laparoscópicas del esófago distal y el estómago. En la mayoría de los casos se utiliza como modelo sin tener en cuenta las características anatómicas específicas de esta especie, por lo que es fundamental realizar un estudio anatómico de la zona del esófago abdominal, cardias y estómago del conejo, que exprese las características y diferencias anatómicas para que los cirujanos estén más familiarizados antes de realizar prácticas laparoscópicas en esta especie. Una diferencia importante es la presencia de una porción grande de esófago abdominal. Está descrito que animales con porciones grandes de esófago abdominal, no tienen la posibilidad de regurgitar o vomitar. Esta diferencia anatómica es muy importante cuando se pretende usar a estos animales como modelo para la enfermedad por reflujo gastroesofágico (ERG).El presente trabajo se realiza con los objetivos de proporcionar a los cirujanos un estudio anatómico que permita conocer la zona del esófago abdominal, cardias y estómago del conejo, para que tengan conocimiento de las características propias en esta especie y las diferencias con el humano, en su uso como modelo animal para la cirugía del cardias y el estómago. Se realizaron medidas anatómicas al esófago y estómago de 18 conejos Nueva Zelanda Blancos de 2.5 a $3.5 \mathrm{Kg}$ de peso de ambos sexos. Donde encontramos que el conejo presenta un una porción grande de esófago abdominal así como un estómago grande con una gran proporción de fundus gástrico.

PALABRAS CLAVE: Conejo; Anatomía comparativa; Esófago; Estómago; Cirugía laparoscópica; Modelos quirúrgicos.

\section{INTRODUCCIÓN}

El conejo pertenece al orden de los lagomorfos (Stevens \& Hume, 1998; Stevens, 2002) y está clasificado como un herbívoro no rumiante con un estómago sencillo. Además, su cavidad abdominal tiene algunas características importantes, como tener un ciego grande que ocupa el $40 \%$ de ella y es 10 veces más grande que el estómago. (Jenkins, 1999; Irlbeck, 2001). Es en este órgano donde el conejo lleva a cabo la fermentación de la fibra vegetal para la obtención de nutrientes (Hume, 2002).

Encontramos poca literatura que describa la anatomía del esófago y estómago del conejo, sobre todo una anatomía encaminada a apoyar al cirujano en la realización de prácticas quirúrgicas cuando el conejo es tomado como modelo animal para la cirugía.
El esófago del conejo presenta 3 capas de músculo estriado, que es semi-voluntario y se extiende dentro del cardias y parte del estómago, y no tiene glándulas mucosas. Además, los conejos no pueden regurgitar, y se ha encontrado que el contacto de sales biliares y acido gástrico producen un severo daño a la mucosa esofágica (Brewer, 2006).

En el humano la capa muscular esofágica es de tipo estriado en el primer tercio, una combinación de estriada y lisa en el medio y solamente músculo liso en la porción más distal (Brewer), pero este concepto está cambiando y en algunos estudios encontraron que el segmento de musculatura estriada es más extenso ( Meyers et al., 1986; Peghini et al., 1998).

* Departamento de Medicina Animal. Universidad de Extremadura, España.

*** Departamento de Ciencias Veterinarias. Universidad Autónoma de Ciudad Juárez, México. 
A diferencia del humano el conejo tiene un cardias con un esfínter bien desarrollado, y por su posición anatómica es imposible que vomiten (Boyd et al., 1960; Islam \& Jain, 2004; Brewer). A este nivel el conejo presenta tanto musculatura lisa como estriada (Botha, 1958).

La mayoría de los reportes de la anatomía del tracto digestivo del conejo están realizados para desarrollar los conocimientos en las áreas de nutrición y de biología. Encontramos por ejemplo que de Oliveira et al., (2001) describen las diferencias de la morfología a nivel histológico del estómago glandular del conejo, encontrando que el estómago no muestra una pared uniforme a lo largo de la circunferencia estomacal, presentando diferencias en las capas serosa, muscular, submucosa y mucosa.

Existen algunos estudios realizados en conejos, en donde se describen que el sistema digestivo de éstos es característico de pequeños herbívoros, que a diferencia de carnívoros u omnívoros como el humano, presentan una capa muscular muy débil en el fundus y cuerpo gástrico, lo que impide el vaciamiento gástrico por motilidad. Para poder vaciar el estómago, el conejo necesita ingerir alimento, para que el alimento recién ingerido desplace al que se encuentra en el estómago, por lo que nunca vamos a encontrar un estómago vacío en un conejo, aun después de ayuno; además el contenido es semisólido, lo que lo hace difícil de vaciar por medio de lavados.

El estómago del conejo representa aproximadamente el 15\% del volumen total del tracto digestivo, pudiendo llegar a pesar $90 \mathrm{~g}$ en un conejo de la raza Nueva Zelanda. El cardias del conejo es una zona grande, relativamente inmóvil con una pared delgada, no glandular y cubierta con un epitelio escamoso estratificado. La capa muscular epicárdica es estriada, siendo una continuación de la del esófago.

Anatómicamente se distinguen dos sectores: la zona del fundus, de paredes finas y que actúa como reservorio y el antro pilórico con mucosa glandular y paredes algo más gruesas. El fundus presenta células parietales y pépticas, siendo la zona del estómago con mayor actividad secretora exocrina. La región pilórica presenta una musculatura fuerte (Brewer).

Una característica del estómago del conejo es que en condiciones de salud, nunca se vacía (Brewer), debido a la escasa musculatura que tiene la mayor parte de la pared. Esto resulta en una mala producción de contracciones necesarias para vaciarlo completamente, es decir, cuando el animal come, el alimento que ingiere llega al estómago, empuja al que había acumulado en él y lo hace pasar a la zona musculada que está próxima al píloro, donde al haber más musculatura se logrará el vaciado (Motta et al., 2006). Está descrita la imposibilidad de vomitar y regurgitar de animales con estómagos que presentan una musculatura débil (Gowen et al., 1999).

La humedad del contenido gástrico oscila entre el 81 y el 83\%, con un pH de alrededor de un 2,5 (Lebas et al., 1986).

El ligamento freno-esofágico es una estructura periesofágica rica en fibras que conecta el esófago con el diafragma, tiene su origen en la fascia de la superficie ventral del diafragma, continuándose después por la fascia transversalis y se inserta cerca de la unión esofagogástrica ( 2 a $3 \mathrm{~cm}$ en el humano). El ligamento forma parte del esfínter esofágico externo y une al esófago con los pilares, usualmente con el derecho (Patnaik \& Mukerjee). Puede considerarse la inserción del ligamento freno-esofágico como la estructura que determina conocer la porción esofágica que está sometida a la presión intraabdominal. A mayor cantidad de esófago abdominal, mayor será la presión abdominal sobre el esófago, por lo que en animales que tienen un esófago abdominal como el humano, la regurgitación es considerada una patología, a diferencia de animales con esófago abdominal reducido, como el perro, donde la regurgitación no siempre es causa de una enfermedad. También se sabe que los animales con mayor proporción de esófago abdominal como la rata, son incapaces de regurgitar o vomitar (Pratschke et al., 2004).

\section{MATERIAL Y MÉTODO}

Para este estudio se utilizaron 18 conejos de la raza Nueva Zelanda, con un peso de 2,5 a 3,5 Kg.

Se realizaron mediciones anatómicas a los 18 conejos para los siguientes parámetros. (Fig. 1).

\section{Esófago:}

- Diámetro del esófago abdominal.

- Longitud del esófago, desde la laringe hasta el cardias.

- Longitud el esófago abdominal, desde el hiato esofágico hasta el cardias. (A)

- Diámetro del esófago al llegar al cardias. (I)

\section{Estómago:}

- Distancia del cardias al fundus. (B)

- Longitud del fundus. (C)

- Distancia de la curvatura mayor a la curvatura menor. (D)

- Longitud del antro. (E)

- Ancho del antro. (F)

- Área del píloro. (G)

- Área del esófago abdominal. (H) 


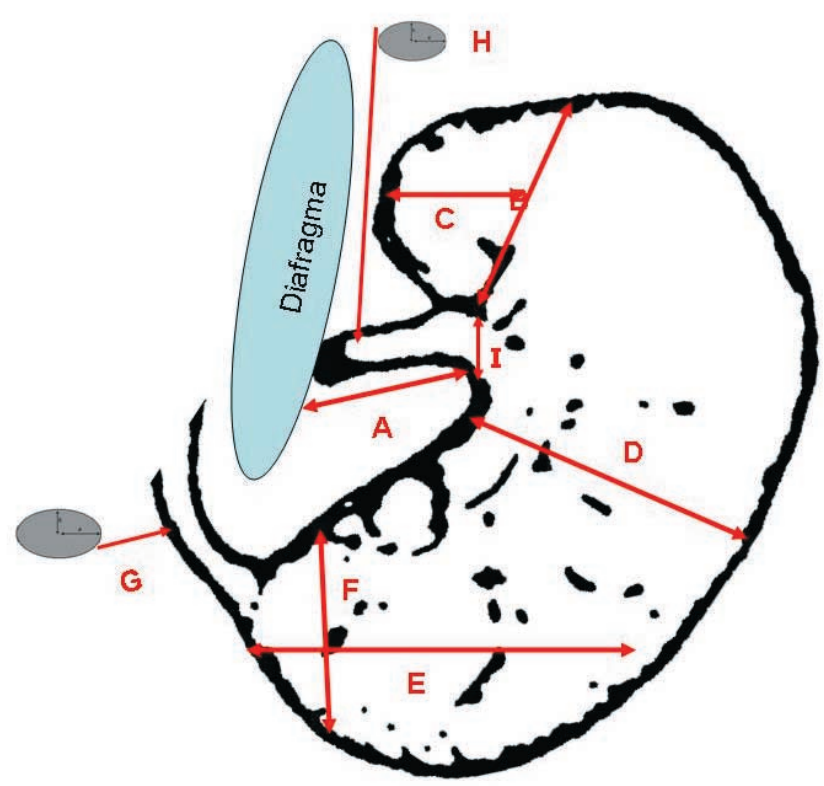

Fig. 1. Diagrama de las mediciones tomadas en el esófago abdominal y el estómago de los conejos.

El largo del esófago abdominal se midió con el estómago y el esófago in situ. (Fig. 2). Las demás medidas fueron realizadas con los órganos fuera del la cavidad abdominal del conejo. Las mediciones fueron realizadas con un calibre (Vernier o pie de rey)

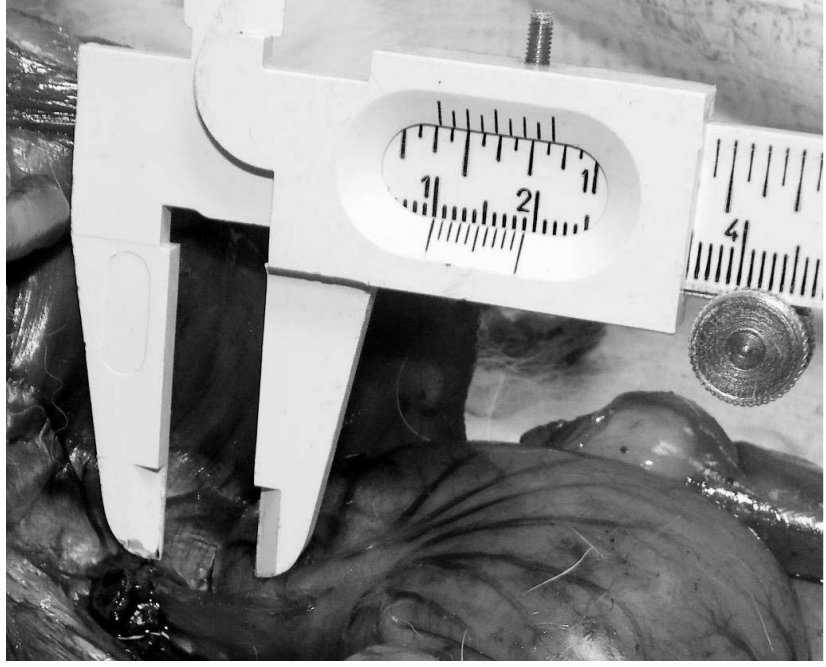

Fig. 2. Medición del esófago abdominal in situ en conejos con el pie de rey.

\section{RESULTADOS}

Las diferentes medidas encontradas en esófagos y estómagos de conejos las podemos observar en la Tabla I.

Tabla I. Estadística descriptiva de las medidas del esófago y estómago en conejos Oryctolagus cuniculus. (Medidas expresadas en centímetros).

\begin{tabular}{|c|c|c|c|c|c|}
\hline & $\mathrm{N}$ & Mínimo & Máximo & Media & Desv. típ. \\
\hline Esófago total & 18 & 14,00 & 17,50 & 16,02 & ,97727 \\
\hline Esófago abdominal (A) & 18 & 1,60 & 2,20 & 1,87 & , 16735 \\
\hline Distancia fundus cardias (B) & 18 & 3,80 & 4,60 & 4,08 & ,20832 \\
\hline Distancia fundus a línea cardias-fundus (C) & 18 & 1,80 & 2,60 & 2,08 & ,21488 \\
\hline Distancia ínter curvaturas (D) & 18 & 3,90 & 6,00 & 5,03 & ,46904 \\
\hline Largo del antro (E) & 18 & 3,40 & 4,40 & 3,93 & ,31060 \\
\hline Ancho del antro (F) & 18 & 2,50 & 3,50 & 3,01 & ,28543 \\
\hline Àrea del píloro (G) & 18 & ,31 & ,94 &, 59 & ,20493 \\
\hline Área esófago (H) & 18 &, 04 &, 11 &, 08 & ,02104 \\
\hline Esófago a la entrada del cardias (I) & 18 &, 80 & 1,40 & 1,09 & ,20164 \\
\hline N válido (según lista) & 18 & & & & \\
\hline
\end{tabular}

\section{DISCUSIÓN}

Esperamos que los resultados obtenidos puedan contribuir en futuras investigaciones donde se utilice al conejo (Oryctolagus cuniculus) como modelo quirúrgico de la región esofágica abdominal y gástrica.
Estamos de acuerdo con Amorim et al., (2001) quienes señalaron que existe poca, escasa e incompleta información sobre el esófago y estómago del conejo (Oryctolagus cuniculus) en tratados de Anatomía Veterinaria, abordándose 
sólo de manera superficial.

Hrislov et al., (2006) presentaron en un estudio de la localización del estómago del conejo en la cavidad abdominal, el cual se encontaría en la porción intratorácica abdominal, la mayor parte de él ocupando la parte izquierda del abdomen y sólo el píloro, se encontaría al lado derecho, a nivel de la $7^{\mathrm{a}}$ costilla, con la que está en contacto. El cardias se sitúa a nivel de la $4^{\mathrm{a}}$ y $5^{\mathrm{a}}$ costillas, un poco más craneal que en algunos otros animales, incluyendo al humano, pues el colon al ser tan grande lo desplaza. La curvatura mayor del estómago desciende ventro-caudalmente y no está en contacto con la pared abdominal. Del lado izquierdo el estómago está en contacto con las costillas $9^{\mathrm{a}}$ a $12^{\mathrm{a}}$ así como con la superficie visceral del hígado. Dorsalmente está en contacto con el diafragma y las porciones proximales de las costillas.

El tamaño del esófago en el conejo $(16 \mathrm{~cm})$ es similar al de un niño, midiendo en los recién nacidos $10 \mathrm{~cm}$ y a los 2 años $16 \mathrm{~cm}$. En el presente estudio encontramos que el conejo tiene una gran porción de esófago abdominal. Estos datos concuerdan con la mayoría de los encontrados en la literatura. Está descrito que esta cantidad de esófago abdominal es una de las causales de la imposibilidad del conejo de regurgitar y vomitar. En términos quirúrgicos, tal cantidad de esófago abdominal es importante, pues es fácilmente localizable y facilita el manejo de él. Además, existe una similitud con la longitud del esófago humano, pues encontramos que el esófago abdominal en los conejos midió 1,87 $\mathrm{cm}$, cantidad grande si lo comparamos con los $3 \mathrm{~cm}$ que mide en un niño (Orestein et al., 2004). Las dimensiones del diámetro del esófago también hacen que sea de fácil lo- calización y manejo quirúrgico. Es importante mencionar la íntima relación que presenta el esófago en su unión con el estómago con los lóbulos hepáticos, sobre todo el izquierdo, lo que podría dificultar su manejo quirúrgico.

Hasta ahora no encontramos artículos que describan las dimensiones del estómago del conejo. Amorim et al., midieron la capacidad volumétrica del estómago de conejos sin raza encontrando que tienen una capacidad de 97,74 ml, sin embargo, estos autores no realizaron medidas de las zonas del estómago. Encontramos que el estómago del conejo presenta un fundus, antro y píloro relativamente grandes. Así, el tamaño del fundus del estómago del conejo permite realizar la mayoría de las endocirugías, como sería la funduplicatura de Nissen. Al igual el tamaño del píloro permite efectuar cirugías de la zona como piloroplastías o piloromiotomías.

Es necesario precaución al realizar cirugías gástricas en conejos, pues al no estar vacío, aún después de ayunos, existe mayor posibilidad de perforarlo.

El tamaño del estómago del conejo permite ser utilizado como modelo quirúrgico para gastronomías, además se podrá utilizar el contenido gástrico como si fuese un cuerpo extraño. La realización de gastrectomías Billroth I y II podrá ser complicada por la presencia de alimento, aunque se debe considerar que el antro pilórico por lo general si está vacío.

Concluimos que el tamaño del esófago abdominal y del estómago del conejo son suficientemente grandes para ser manejados quirúrgicamente en laparoscopía.

RODRÍGUEZ-AlarCón, C.; PÉREZ, E.; MARTÍN, U.; RIVERA, R.; HERNÁNDEZ, A.; VIVO, J.; BERISTAIN, M. \& USÓN, J. Morphometry of the abdominal esophagus and stomach of the rabbit. Laparoscopyc surgery applications. Int. J. Morphol., 28(1):27-31, 2010.

SUMMARY: Actuality, there are few studies about rabbits (Orycotolagus cuniculus) digestive tract anatomy and physiology. These studies are designed at nutrition and biology of the rabbit. But few deal with the anatomy of a surgeon's vision. There are also some researches that propose rabbits as laparoscopic surgery animal model for of the distal oesophagus and stomach. In most cases is used as a model without considering the specific anatomical characteristics of this species. So it is essential to make an anatomical study of the abdominal oesophagus, cardia, and stomach of the rabbit. This should express the characteristics and anatomical differences between species. On the way to that surgeon are more familiar before performing laparoscopic practices in this species. An important difference is the presence of a large portion of abdominal oesophagus. There is reported that animals with large portions of abdominal oesophagus, can not vomit or regurgitate. This anatomical difference is very important when trying to use these animals as a model for gastroesophageal reflux disease. This work is done with the objectives of providing surgeons an anatomical study of the abdominal esophagus, cardia and stomach of the rabbit, to have knowledge of the characteristics in this species and the differences with humans, when used as surgical animal model. Anatomical measures were made to the oesophagus and stomach of 18 males and females New Zealand White rabbits. Between 2,5 to $3,5 \mathrm{Kg}$. We find that the rabbit has a large portion of an abdominal oesophagus and a large stomach with a large proportion of gastric fundus.

KEY WORDS: Rabbit; Comparative anatomy; Esophagus; Stomach; Laparoscopic surgery; Surgical models. 


\section{REFERENCIAS BIBLIOGRÁFICAS}

Amorim, A. M. J.; Amorin Jr., A. A.; Villarouco, de O. F. M.; Ojea B. D. \& Costa, F. D. J. F. The stomach capacity of underbred rabbits (Oryctolagus cuniculus). Rev. chil. Ana., 19(3):259-62, 2001.

Botha, G. S. M. A note on the comparative anatomy of the cardioesophageal junction. Acta Anatomica, 34:52, 1958.

Boyd, C. E.; Boyd, E. M. \& Brown, M. D. Penicillin vomiting. Can. Med. Assoc. J., 82(4):195-7, 1960.

Brewer N. Biology of the rabbit. J. Am. Assoc. Lab. Anim. Sci., 45:8-24, 2006.

de Oliveira, L. R.; Molinari, S. L.; Natali, M. R. M.; Michelan, A. C. \& Scapinello, C. Morphologic considerations about the wall of the glandular stomach of young rabbits (Oryctolagus cuniculus). Rev. chil. Anat., 19(3):253-258, 2001.

Gowen, G. F.; Stoldt, H. S. \& Rosato, F. E. Five Risk Factors Identify Patients with Gastroesophageal Intussusception. Arch Surg., 134:1394-1397, 1999.

Hristov, H.; Kostov, D. \& Vladova, D. Topographical anatomy of some abdominal organs in Rabbits. Trakia Journal of Sciences., 4(3):7-10, 2006.

Hume, I. D. Digestive strategies of mammals. Acta Zoologica Sinica,. 48(1):1-19, 2002.

Irlbeck, N. A. How to feed the rabbit (Oryctolagus cuniculus) gastrointestinal tract. J. Anim. Sci., 79:343-6, 2001.

Islam, S. \& Jain, P. N. Post-operative nausea and vomiting (PONV): a review article. Indian J Anaesth., 48(4):25358, 2004.

Jenkins, J. R. Feeding Recommendations for the House Rabbit. Veterinary Clinics of North America: Exotic Animal Practice., 2:143-151, 1999.

Lebas, F.; Coudert, P.; Rouvier, R. \& de Rochambeau, H. The Rabbit:Husbandry, Health and Production, FAO Animal Production and Health Series no. 21, FAO, Roma, 1986. www.fao.org/docrep/x5082e/x5082e00.htm .

Meyer G. W.; Austin R. M.; Brady C. E. \& Castell D. O. Muscle anatomy of the human esophagus. J. Clin Gastroenterol., 8(2):131-4, 1986
Motta, W. F.; Borges, F. M. \& Apocaypse, R. Fundamentos da nutrição de coelhos. Memorias del III Congreso de Cunicultura de las América. Paraná. Brasil, 2006.

Orenstein, S.; Peters, J.; Khan, S.; Youssef J. \& Zahed, S. El esófago. En: Richard E. Behrman, Robert M. Kliegman, Hal B. Jenson (Ed.). Nelson Tratado de pediatría. 17 . España, Elsevier, 2004. pp. 1217

Patnaik, V. V. G. \& Mukerjee, R. N. Role of PhrenoEsophageal Membrane And Mucosal Flaps in the Functioning of the Gastroesophageal Junction Morphologic And Microanatomic Study In Mammals Including Man. J. Anat. Soc. India, 49(1):31-9, 2000.

Peghini P. L.; Pursnani K. G.; Gideon M. R.; Castell J. A.; Nierman J.; \& Castell D. O. Proximal and distal esophageal contractions have similar manometric features. Am. J. of Physiology, 274: 325-30, 1998.

Pratschke, K. M.; Fitzpatrick, E.; Campion, D.; McAllister, H. \& Bellenger, C. R. Topography of the gastrooesophageal junction in the dog revisited: possible clinical implications. Research in Veterinary Science., 76:171-7, 2004.

Stevens, C. E. \& Hume, D. Contributions of Microbes in Vertebrate Gastrointestinal Tract to Production and Conservation of Nutrients. Physiol. Rev., 78:393-427, 1998.

Stevens C. E. Digestive System of Mammals. Encyclopedia of life sciences., 1-8, 2002.

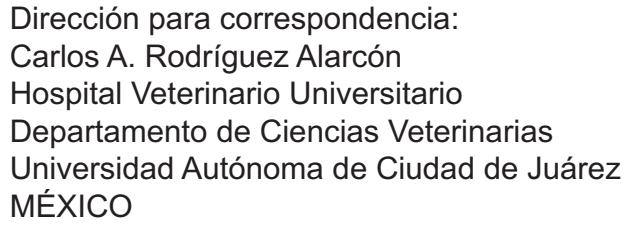

Email: mvzcara@hotmail.com

Recibido : 29-06-2009

Aceptado: 14-02-2010 
